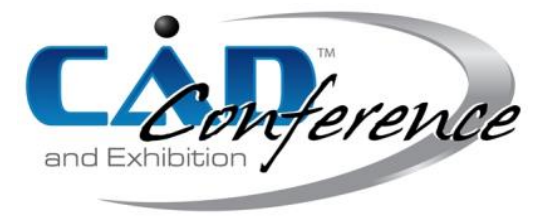

Title:

Generative Computer-Aided Design: New Design Tool for Direct Physical Production

Authors:

Lujie Chen, chenlujie@sutd.edu.sg, Singapore University of Technology and Design, Singapore

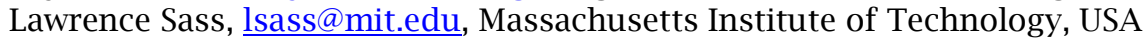

Keywords:

Generative CAD, Rapid prototyping, Contouring, Plate forming, Direct physical production

DOI: 10.14733/cadconfP.2016.1-4

Introduction:

Computer-Aided Design has come a long way since the advent of computer technology. Design, a human-centered cognitive activity, has been facilitated, advanced, and even partly replaced by computers through the development of sophisticated numerical algorithms. Computer technology that enables design is a consequence of observation and extraction of repeated, replicable work embedded a design process. In early days, such replicable work pertained to drafting, printing and error checking. Now, deeper observation of the design process has motivated engineers to create computerbased design tools that are able to accomplish highly complicated work without human intervention. The trend of offloading replicable work from designers to computers leads to Generative CAD, an approach that will have considerable impact in design and manufacturing fields.

Today, heavy industries such as civil construction and ship building usually have their design and manufacturing processes implemented in four phases (e.g. [1,2]) (Fig. 1).
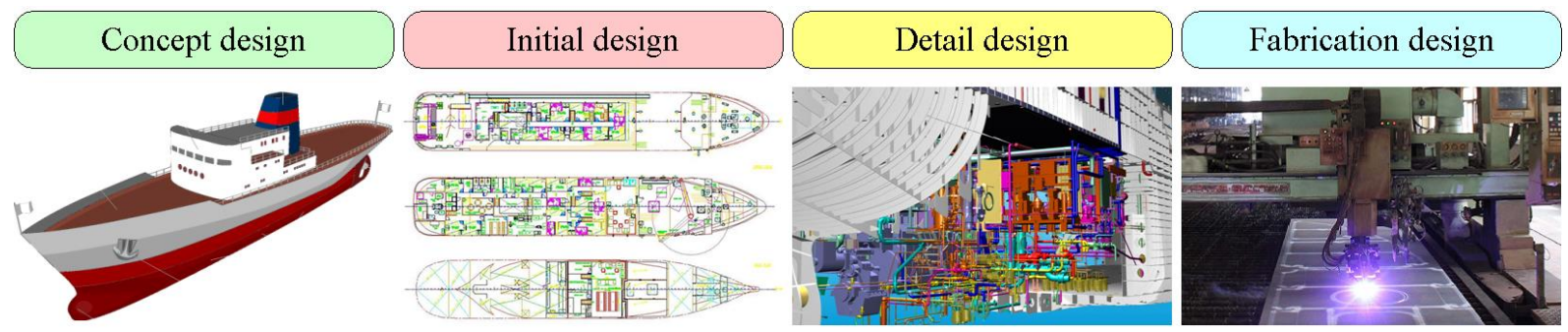

Fig. 1: Typical four design phases of heavy industries.

The first phase is called basic or concept design, in which aesthetics and basic functions of a product are defined in digital models. Quantification of size, weight, material, etc. of the product is loosely addressed with little detail. Companies involved in the concept design are architecture firms and design studios. The second phase is initial design, where the conventional engineering design is involved. Questions related to number of rooms in a building, maximum load of a ship, etc. are addressed, and major dimensions, weight and material of the product are quantified. Companies involved in the initial design are construction firms and ship design companies who have specific domain knowledge of the industry. The third phase is detail design, in which all parts of the production are modelled in detail. Typically, lots of engineers are required in the work using conventional CAD tools to do drafting, measurement, calculation and error checking. Companies involved are still construction firms and ship design companies; or sub-contractors of these firms. The 
last phase is fabrication design, where the fabrication method of each component is determined, and the components are manufactured. Companies involved are mostly fabrication-oriented workshops, pre-fabrication companies, metal cutting service providers, or manufacturing department of ship builders.

Even though conventional CAD tools and automated machines have been extensively utilized in the process, heavy industries are still faced with problems such as lack of manpower, complexity in transition between design phases, and waste of material due to distributed manufacturing facilities. Today, these problems are prominent in developed countries. Tomorrow, they will become an issue worldwide that awaits for a solution.

In this paper, we report our investigation on a Generative CAD approach called Direct Physical Production (DPP), where parts of physical artifacts are directly generated by computer algorithms from input digital models $[3,4]$. The approach bypasses human intervention in drafting and modeling the parts of a product, and fabrication-oriented data are generated automatically ready for manufacturing. We demonstrate that novel methods in this approach can produce scalable products potentially useful for architecture, construction, and various industries.

\section{Direct Physical Production:}

In the context of this work, DPP pertains to producing a physical artifact directly from a digital model. Human intervention is minimized in generation of model parts but is required during model assembly. At present our work aims at creating new design tools for low-fidelity rapid prototyping; in long term, the methods developed will be applicable to producing functional products for end users.

Three methods are introduced: one-dimensional (1D) contouring, 2D contouring, and plate forming. It is assumed that the input digital model is a 3D object in a triangle mesh format. Each method generates a specific physical representation of the object.

\section{D Contouring:}

Given a triangle mesh object in 3D, it is possible to extract 1D contours by intersecting the mesh with parallel planes, as shown in Figs. 2(a) and 2(b). Each contour may be treated as a planar sheet; its shape in 2D can be fabricated by laser cutting. The assembly of the contours produces a discrete representation of the object [Figs. 2(c) and 2(d)].
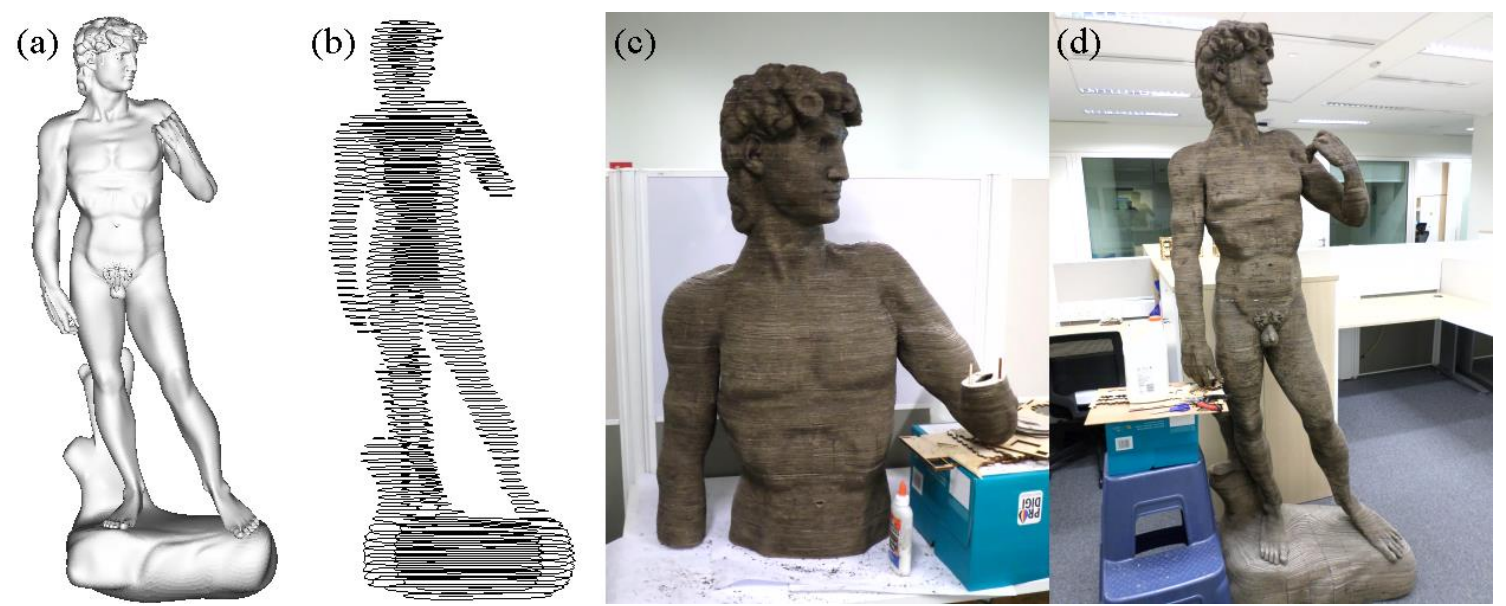

Fig. 2: Stature produced by 1D contouring method. (a) Input digital model. (b) 1D contours extracted by a computer program. (c) Assembled bust. (d) Assembled physical model consisting of 5425 parts.

If the size of a contour is larger than the working area of a laser cutter, the contour can be partitioned into smaller parts. To save material, a contour may also be hollowed by generating an 
inner rim. The stand and upper body of the stature is hollow, as indicated in Figs. 2(b). Assembly is achieved by sticking adjacent contours. Alignment is critical during assembly; it is facilitated by automatically generated reference holes in each contour.

\section{D Contouring:}

2D contours can be extracted from a triangle mesh by intersecting the mesh with orthogonal planes, as shown in Figs. 3(a) and 3(b). The assembly of a physical model is enabled by interlocking the contours in two directions using automatically generated slots [Fig. 3(c)]. Size of the slots is determined by the local geometry of the mesh; thickness of the slots is determined by that of the cut sheet. A tolerance setting may be left for a user to adjust. Larger tolerance leads to relatively wide slots, while smaller tolerance leads to tight slots. The tolerance should be determined from material in use (e.g. cardboard, plywood, plastic) and laser cutting settings (e.g. speed, power).
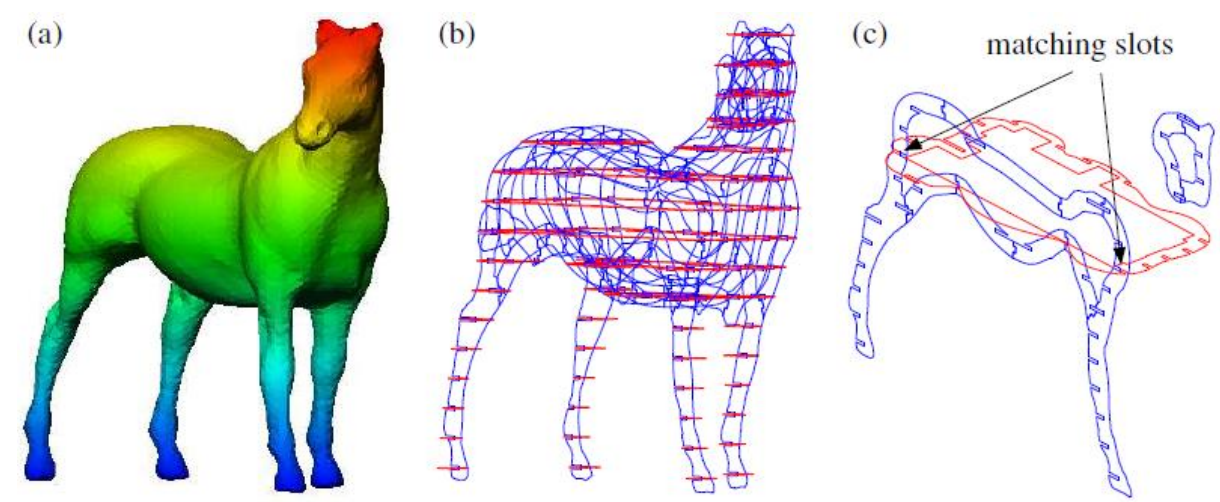

Fig. 3. (a) A horse model. (b) 2D contours. (c) Slots for interlocking the contours.

\section{Plate Forming:}

Plate forming is most suitable in making objects comprised of planar faces. An edge shared by two faces is generated with finger joints for interlocking the faces. The physical model is a watertight representation of the object in contrast to the discrete representation produced by the $1 \mathrm{D}$ and $2 \mathrm{D}$ contouring methods.

Because of the finger joints, assembly of the physical model is relatively easy, which enables physical production of highly complex objects, such as the cube shown in Fig. 4 The end object may be used as mold for material casting due to being watertight. A potential application of the method is to create formwork for concrete casting, a process that is time consuming, low accuracy, and labor demanding in current industry practice.
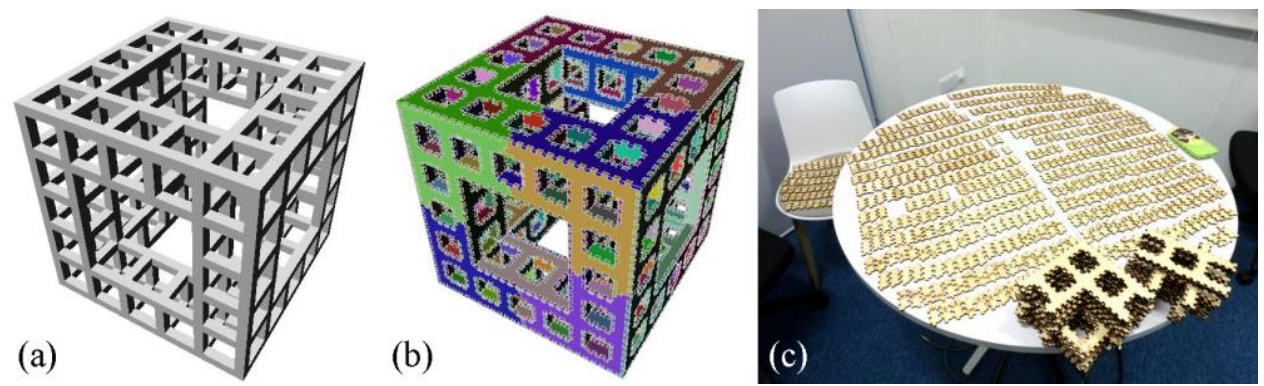

Proceedings of CAD'16, Vancouver, Canada, June 27-30, 2016, 1-4 (c) 2016 CAD Solutions, LLC, http://www.cad-conference.net 


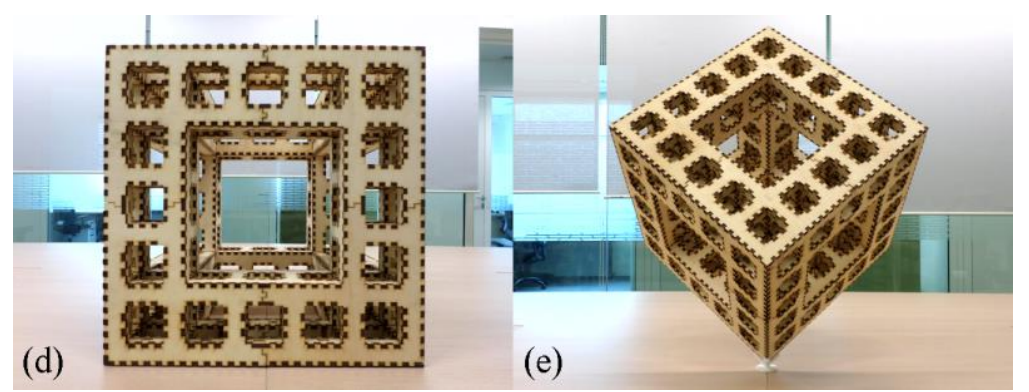

Fig. 4: (a) A cube model. (b) Planar parts. (c) Laser cut plates. (d, e) Assembled cube.

\section{Conclusion:}

Three methods are introduced to directly produce physical artifacts from digital models. Human intervention is minimized in the process of modelling parts and generating fabrication data. The approach, named as Generative $\mathrm{CAD}$, aims at extracting high-level of replicable work in a design process, and free designers and builders to focus on more complicated tasks that require human's cognitive ability irreplaceable by machines nowadays. Deeper investigation of Generative CAD methods will create viable solutions to design and manufacturing industries that heavily relies on manpower.

\section{References:}

[1] Neptun Ship Design: http://www.neptun-germany.com/design-phases/

[2] Scherer Construction: http://schererconstruction.com/services/

[3] Schulz, A.; Shamir, A.; Levin, D. I. W.; Sitthi-amorn, P.; Matusik, W.: Design and fabrication by example, ACM Trans Graph., 33(4), 2014, 62:1-62:11. http://dx.doi.org/10.1145/2601097.2601127

[4] Yuliy, S.; Pauly, M.: Fabrication-ware design with intersecting planar pieces, Comput Graph Forum 32(2), 2013, 317-26. http://dx.doi.org/10.1111/cgf.12051 